\title{
Effect of Midodrine in Patients with Liver Cirrhosis and Refractory Ascites
}

\author{
Ahmed A. Obiedallah ${ }^{*}$, Essam Abdelmohsen, Abdalla I. Kelani, Mohamed Mousa \\ Department of Internal Medicine, Faculty of Medicine, Assiut University, Assiut City, Egypt \\ Email address: \\ ahmed_obiedallah@yahoo.com (A. A. Obiedallah), essam_223@yahoo.com (E. Abdelmohsen), Abdallakelani@yahoo.com (A. I. Kelani), \\ Mohamedmousa5544@gmail.com (M. Mousa) \\ ${ }^{*}$ Corresponding author
}

\section{To cite this article:}

Ahmed A. Obiedallah, Essam Abdelmohsen, Abdalla I. Kelani, Mohamed Mousa. Effect of Midodrine in Patients with Liver Cirrhosis and Refractory Ascites. American Journal of Internal Medicine. Vol. 5, No. 1, 2017, pp. 12-17. doi: 10.11648/j.ajim.20170501.13

Received: January 1, 2017; Accepted: January 20, 2017; Published: February 20, 2017

\begin{abstract}
Background: Ascites is the most common complication of liver cirrhosis and about 5-10\% of all cases develop refractory ascites, $50 \%$ of such patients die within 6 months of its development. Aim of the Work: to assess the usefulness of adding midodrine beside the standard medical treatment for patients with liver cirrhosis and refractory ascites. Patients and Methods: This study included 78 patients with liver cirrhosis and refractory ascites or recurrent ascites, Group A: (n=37) patients on standard medical treatment (SMT) [low sodium diet + diuretic therapy (loop diuretic in a dose 40-160 mg/day and distal acting diuretic in a dose 100-400 mg/day + large volume paracentesis as needed] Group B: $(\mathrm{n}=41)$ patients with standard medical treatment (SMT) and midodrine tolerable dose. Results: Statistical significant difference between the SMT group and midodrine group as regard reduction in body weight and increase in mean arterial blood pressure and 24 h-urinary volume where $\mathrm{P}$ value was $<0.05$. Conclusion, midodrine is a safe treatment for patients with liver cirrhosis and its addition to standard medical treatment is associated with better control of ascites.
\end{abstract}

Kerywords: Midodirine, Refractory Ascites, Liver Cirrhosis

\section{Introduction}

Ascites is the most common complication of cirrhosis. Once ascites develops, the prognosis worsens and the patient becomes susceptible to complications such as bacterial peritonitis, hepatic hydrothorax, hyponatraemia, and complications of diuretic therapy [1].

Following the development of ascites, the patient's quality of life declines significantly and survival is about $50 \%$ after 5 years of follow-up [2]. Once the ascites becomes refractory to medical therapy, the prognosis is worse, with only $40-60 \%$ of patients alive after 2 years without liver transplantation [3].

The pathophysiology of ascites is complex, and various mechanisms have been proposed. Splanchnic and systemic vasodilatation related to excess nitric oxide has been associated with systemic hypotension and increased portal flow. This is associated with hemodynamic compensatory mechanisms via activation of the renin-angiotensin- aldosterone and sympathetic nervous systems and the nonosmotic release of antidiuretic hormones [4].

Refractory ascites develops in approximately $5-10 \%$ of all cases of ascites and $50 \%$ of such patients die within 6 months of its development. Portal hypertension and splanchnic vasodilatation causes more marked arterial under filling and the sodium retaining mechanisms become permanently activated [5]. The therapeutic options available for patients with refractory ascites are serial therapeutic paracentesis, liver transplantation, transjagular intrahepatic portosystemic shunts (TIPS), and peritoneovenous shunt [6].

Several studies have evaluated the efficacy of various vasopressors in patients with hepatorenal syndrome [7], [8], [9]. Vasoprssors have also been used for prevention of postparacentesis circulatory dysfunction with variable results [10] [11].

Recently, various vasoconstrictors have been used in 
nonazotemic cirrhotic patients with ascites and showed an improvement in circulatory and renal function and sodium excretion [12], [13].

Midodrine, an alpha-1 agonist acting directly on the peripheral alpha-receptors, has been widely used in the therapy of orthostatic hypotension and various secondary hypotensive disorders. It has recently been shown that singledose administration of midodrine leads to a significant improvement in systemic and renal hemodynamics in nonazotemic cirrhotic patients with ascites, the beneficial effects of midodrine have been attributed to its modulating effects on autonomic function and increased peripheral blood vessel resistance [4].

Midodrine has been found to decrease nitrite and nitrate activity in patients with ascites with or without HRS who had decreased plasma renin activity and decreased levels of antidiuretic hormone [14], This could be a possible mechanism for decreasing portal pressure and decreasing ascitic fluid accumulation. There is also evidence that a similar reduction in fluid accumulation may occur with use of vasoconstrictors in patients with end-stage liver disease without a significant renal function improvement [15].

Angeli et al. reported that $15 \mathrm{mg}$ of oral midodrine increased renal plasma flow by $40 \%$, GFR by $21 \%$ and urinary sodium excretion by $28 \%$. When combined, the studies by Villeneuve et al. and Angeli et al. suggest that diuretic resistance in cirrhotic patients with ascites is possibly due to diminished GFR, and orally administered midodrine could significantly improve GFR in nonazotemic cirrhotics with ascites [15].

Midodrine along with octerotide and albumin, has been shown to better control of ascites in a short term pilot study in patients with refractory ascites [15].

\section{Aim of the Work}

To study the usefulness of adding midodrine beside the standard medical treatment for patients with liver cirrhosis and refractory ascites.

\section{Patients \& Methods}

This study was conducted in the hepatology and gastroenterology unit and outpatients' clinic in internal medicine department, Assiut University.

Consent was taken from all patients involved in this study with the approval of Ethical Committee of faculty of medicine, Assiut University.

This study included 90 patients with liver cirrhosis and refractory ascites or recurrent ascites. Cirrhotic patients were enrolled according to having altered Child-Pough score and / or ultrasonographic finding of cirrhosis.

* Refractory ascites defined according to the report of the consensus conference of the International Ascites Club in 2003 as ascites that cannot be mobilized or the early recurrence of which (i.e. after therapeutic paracentesis) cannot be satisfactorily prevented by medical therapy and It is divided into: a) Diuretic-resistant ascites: ascites that cannot be mobilized or the early recurrence of which cannot be prevented because of a lack of response to dietary sodium restriction and intensive diuretic treatment and b) Diureticintractable ascites: ascites that cannot be mobilized or the early recurrence of which cannot be prevented because of the development of diuretic-induced complications that preclude the use of an effective diuretic dosage.

The patients were divided into 2 groups

Group $A$ : $(\mathrm{n}=45)$ patients on standard medical treatment [low sodium diet + diuretic therapy (loop diuretic in a dose $40-160 \mathrm{mg} /$ day and distal acting diuretic in a dose 100-400 $\mathrm{mg} /$ day + large volume paracentesis as needed] 8 were excluded during the screening period for various reasons (spontaneous bacterial peritonitis in 2, encephalopathy in 1, acute kidney injury in 2 , hepatorenal syndrome in 1 , and gastrointestinal bleeding in 2 patients).

Group B: $(\mathrm{n}=45)$ patients with standard medical treatment (SMT) and midodrine tolerable dose. 4 were excluded during the screening period

(spontaneous bacterial peritonitis in 1 , encephalopathy in 1 , and gastrointestinal bleeding in 2 patients).

All patients were subjected to:

I. Full history taking regarding age, sex, etiology of cirrhsis, duration of disease, other comorbidity and medications.

II. Meticulous examination and assessment for the following before and after one month of treatment with midodrine in tolerable dose

a. Mean arterial blood pressure [diastolic blood pressure $+1 / 3$ pulse pressure or $2 / 3$ diastolic blood pressure $+1 / 3$ systolic blood pressure.

b. Weight.

c. Frequency of indicated large volume paracentesis paracentesis (LVP) $(>50 \mathrm{ml} / \mathrm{kg}$ of ascites) and volume removed each time.

* Therapeutic paracentesis is indicated for the following:

- Respiratory compromise secondary to ascites

- Abdominal pain or pressure secondary to ascites (including abdominal compartment syndrome).

d. d-Glomerular filtration rate by MDRD (Modification of Diet in Renal disease) equation.

e. e-Volume of urine in 24-hour

f. f-MELD score (Model for End Stage Liver Disease)

g. g-Child-Pough classification score.

h. h-ECG and Echocardiogarphy (all patients that have coronary artery disease, valvular heart disease with LV systolic dysfunction or cardiomyopathy were excluded)

The following investigations were done: Complete Blood Counts, Liver function test, coagulation profile (prothrombin time and concentration and INR), kidney function tests, ascetic fluid study,

Urine analysis, creatinine clearance.

Statstical Analysis

Statistical analysis was conducted using Statistical 
Package for Social Science version 16.0 for Windows software (SPSS Inc.) Qualitative variables (all Categorical variables) were expressed as frequency and percentage (N, \%). Chi-square test was used to compare qualitative variables. Quantitative variables were presented in terms of, mean \pm standard deviation (Mean, SD).

For quantitative data, comparison between two groups was done using Student's T-test. Level of significance "P" value was evaluated, where $\mathrm{P}$ value $<0.05$ was considered statistically significant.

\section{Results}

Our study included 78 patients diagnosed to have liver cirrhosis and refractory ascites were admitted in gastroenterology unit and gastroenterology outpatients' clinic in internal medicine department, Assiut University. Those patients were divided into 2 groups:

- Group 1: patients treated with standard medical treatment $(n=37)$ (SMT group) (on low sodium diet, diuretics therapy and paracentesis if needed)

- Group 2: patients treated with SMT plus midodrine in maximum tolerable dose group $(\mathrm{n}=41)$.

Part 1: Demographic data of the studied patients

The demographic data of the SMT group showed that the mean of patients' age was $55 \pm 12$ vs. $58 \pm 9$ for the midodrine group. Male to female distribution was 9:1 for the SMT vs. 5:1 for the midodrine group. Liver cirrhosis was secondary to mainly HCV and HBV infection in both groups. Mean of the duration of cirrhosis was $4 \pm 2$ and $3.5 \pm 2$ years for SMT group and midodrine group respectively

As regard the demographic data; there was no statistical significant difference between both group where $P$ value $>0.05$.
Table 1. Demographic data of the studied patients.

\begin{tabular}{llll}
\hline Variables & $\begin{array}{l}\text { SMT group } \\
(\mathbf{n}=\mathbf{3 7})\end{array}$ & $\begin{array}{l}\text { Midodrine } \\
\text { group (n=41) }\end{array}$ & P value \\
\hline Age & $55 \pm 12$ & $58 \pm 9$ & 0.09 \\
Sex & $33(89.2 \%)$ & $34(82.9 \%)$ & \\
Male & $4(10.8 \%)$ & $7(17.1 \%)$ & 0.43 \\
Female & & & \\
Aetiology of cirrhosis & $28(75.7 \%)$ & $31(75.6 \%)$ & \\
HCV & $9(24.3 \%)$ & $7(17.1 \%)$ & 0.36 \\
HBV & 0 & $2(4.9 \%)$ & 0.81 \\
Autoimmune & 0 & $1(2.1 \%)$ & \\
Other causes & $4 \pm 2$ & $3.5 \pm 2$ & \\
Duration of cirrhosis & & & \\
Co-morbidities & & $27(65.9 \%)$ & \\
Nothing & $24(64.9 \%)$ & 0.08 & \\
Diabetes mellitus & $9(24.3 \%)$ & $4(10.8 \%)$ & \\
Heart diseases & & & \\
\hline
\end{tabular}

Chi square test was used

Part 2: Clinical parameters before and one month after therapy for both groups

For both group weight, mean arterial blood pressure and heart rate were followed basal and one month after therapy. There was significant statistical difference between both groups as regard reduction in body weight and increase in mean arterial blood pressure where $P$ value was 0.00 and 0.04 respectively. In contrast there was no statistical significant difference between heart rate in both groups $(\mathrm{P}$ value $>0.05)$.

Table 2. Clinical parameters before and one month after therapy for both groups.

\begin{tabular}{|c|c|c|c|c|c|c|c|}
\hline \multirow{2}{*}{ Variables } & \multicolumn{2}{|c|}{ SMT group $(n=37)$} & \multirow{2}{*}{$\begin{array}{l}\text { Mean } \\
\text { change } \pm \text { SD }\end{array}$} & \multicolumn{2}{|c|}{ Midodrine group $(n=41)$} & \multirow{2}{*}{$\begin{array}{l}\text { Mean } \\
\text { change } \pm S D\end{array}$} & \multirow{2}{*}{ P. value ${ }^{\#}$} \\
\hline & Basal & After one month & & Basal & After one month & & \\
\hline Weight (kg) & $76.2 \pm 11.4$ & $74.9 \pm 10.7$ & $(-1.3) \pm 0.8$ & $77.2 \pm 9.5$ & $72.7 \pm 8.4$ & $(-4.5) \pm 1.1$ & $0.00 *$ \\
\hline Mean arterial blood pressure $(\mathrm{mmHg})$ & $78.6 \pm 12.1$ & $80.8 \pm 11.7$ & $2.2 \pm 1.3$ & $77.7 \pm 11.1$ & $86.2 \pm 11.6$ & $8.5 \pm 2.1$ & $0.00 *$ \\
\hline Heart rate $(\mathrm{b} / \mathrm{m})$ & $89.8 \pm 9.3$ & $88.4 \pm 10.1$ & $(-1.4) \pm 0.7$ & $88.1 \pm 9.1$ & $86.9 \pm 10.8$ & $(-1.2) \pm 0.3$ & 0.08 \\
\hline
\end{tabular}

\# Comparison between mean changes in studied groups (independent sample t test was used)

Part 3: History of therapeutic paracentesis and drugs dosage and its side effects

Therapeutic paracentesis was done in $11 / 20$ patients with SMT vs. $45 / 60$ patients from the midodrine group. Paracentesis was followed by replacement salt free albumin in eight patients with SMT and 22/45 patient from the midodrine group. Mean of the removed amount was $3 \pm 0.5$ and $3.5 \pm 1$ for both groups respectively. No statistical difference between both groups as regard therapeutic paracentesis and albumin replacement.

Midodrine dose reached upto $15 \mathrm{mg} /$ day according the tolerability by the patients with mean $12.5 \pm 2.5 \mathrm{mg}$ without any significant difference while mean of loop diuretics was $120 \pm 20$ and $120 \pm 10$ for SMT and midodrine group respectively. Mean of $\mathrm{K}$ sparing diuretics was $300 \pm 50 \mathrm{mg}$ for SMT group and $300 \pm 100 \mathrm{mg}$ for midodrine group. Side effects of diuretics therapy were noticed in 20 patients ( 7 from SMT and 13 from midodrine groups). There was statistical significant difference between both group in dosage of diuretics or midodrine where $\mathrm{P}$ value was $>0.05$. 
Table 3. History of therapeutic paracentesis and drugs dosage and its side effects.

\begin{tabular}{lll}
\hline Variables & SMT group $(\mathbf{n = 3 7})$ & Midodrine group (n=41) \\
\hline History of therapeutic paracentesis & & P value \\
Yes & 20 & 30 \\
No & 17 & 11 \\
Amount removed (1/time) (mean \pm SD) & $3 \pm 0.5$ & $3.5 \pm 1$ \\
Replacement with salt free albumin & & 15 \\
Yes & 14 & 15 \\
No & 6 & 0.08 \\
Drug dosage & & $12.5 \pm 2.5$ \\
Midodrine & $0 \pm 0$ & $120 \pm 10$ \\
Loop diuretics & $120 \pm 20$ & $300 \pm 100$ \\
K- sparing diuretics & $300 \pm 50$ & 0.16 \\
Side effect of the diuretics & & 12 \\
Yes & 12 & 29 \\
No & 25 & 0.67 \\
\hline
\end{tabular}

Chi square test was used

Part 4: Laboratory parameters before and one month after therapy for both groups.

For both groups the following investigations were done; CBC, LFTs, coagulation profile, BUN, serum creatinine, glomerular filteration rate, as well as Child score and MELD score were calculated. Twenty four urinary volumes were estimated for both groups before and one month after therapy.

For all the laboratory parameters; there was only statistical significant difference between both groups as regard twenty four urinary volumes at start of therapy and one month later on where $\mathrm{P}$ value was 0.04 .

Table 4. Laboratory parameters before and one month after therapy for both groups.

\begin{tabular}{|c|c|c|c|c|c|}
\hline \multirow{2}{*}{ Variables } & \multicolumn{2}{|c|}{ SMT group $(n=37)$} & \multicolumn{2}{|c|}{ Midodrine group $(n=41)$} & \multirow{2}{*}{ P value } \\
\hline & Basal & After one month & Basal & After one month & \\
\hline Hemoglobin (g \%) & $11 \pm 1.3$ & $10 \pm 1.9$ & $11 \pm 1.01$ & $10 \pm 2.1$ & 0.09 \\
\hline Platelets (x 103/1) & $90 \pm 13$ & $88 \pm 15$ & $84 \pm 15$ & $83 \pm 13$ & 0.54 \\
\hline PT (seconds) & $13 \pm 3$ & $14 \pm 2$ & $15 \pm 3$ & $14 \pm 2$ & \\
\hline $\mathrm{PC}(\%)$ & $55 \pm 8$ & $51 \pm 9$ & $49 \pm 8$ & $50 \pm 6$ & \\
\hline INR & $1.12 \pm .44$ & $1.22 \pm .54$ & $1.2 \pm .34$ & 1.12 & 0.98 \\
\hline ALT (U/1) & $87 \pm 15$ & $89 \pm 19$ & $57 \pm 15$ & $89 \pm 13$ & 0.99 \\
\hline AST (U/1) & $111 \pm 10$ & $123 \pm 9$ & $101 \pm 11$ & $121 \pm 11$ & 0.65 \\
\hline Albumin (g/l) & $2.54 \pm 0.98$ & $2.43 \pm 0.52$ & $2.1 \pm 0.93$ & $2.09 \pm 0.2$ & 0.86 \\
\hline Total protein $(\mathrm{g} / \mathrm{l})$ & $5.56 \pm 0.18$ & $5.46 \pm 0.43$ & $4.89 \pm 0.23$ & $5.11 \pm 0.22$ & 0.31 \\
\hline Creatinine (mg\dl) & $1.02 \pm 0.42$ & $1.3 \pm 0.11$ & $1.1 \pm 0.5$ & $0.9 \pm 0.12$ & 0.53 \\
\hline Urea $(\mathrm{mg} / \mathrm{dl})$ & $4 \pm 3$ & $5 \pm 3$ & $4 \pm 2$ & $5 \pm 2$ & 0.98 \\
\hline Urine volume $(\mathrm{ml} / 24 \mathrm{~h})$ & $1198 \pm 299$ & $1277 \pm 313$ & $1192 \pm 287$ & $1376 \pm 301$ & $0.00 *$ \\
\hline GFR (ml/min) & $99 \pm 34$ & $89 \pm 40$ & $112 \pm 22$ & $109 \pm 38$ & 0.12 \\
\hline MELD score & $14 \pm 2$ & $15 \pm 3$ & $17 \pm 2$ & $16 \pm 2$ & 0.14 \\
\hline
\end{tabular}

From our study there were statistical significant differences between the SMT group and midodrine group as regard reduction in body weight and increase in mean arterial blood pressure and $24 \mathrm{~h}$-urinary volume where $\mathrm{P}$ value was $<$ 0.05 .

\section{Discussion}

Ascites is the most frequent complication of cirrhosis, occurring in nearly $50 \%$ of patients within 10 years after cirrhosis is diagnosed [4]. Ascites is the most common cause of hospital admissions in cirrhotic patients and the development of ascites predicts a mortality of approximately $15 \%$ and $44 \%$ at 1 and 5 years, respectively [16].

Refractory ascites develops in approximately $5-10 \%$ of all cases of ascites and $50 \%$ of such patients die within 6 months of its development, A proportion of these patients require large-volume paracentesis (LVP) for symptom relief when other treatment modalities are unsuccessful or impossible. However, frequent LVP is associated with patient discomfort, decreased quality of life, and increased potential risks, such as circulatory dysfunction and related complications [17].

Since splanchnic arterial vasodilatation is a constant feature of cirrhosis, arterial vasoconstrictors could be of value in cirrhotic patients. Administration of (midodrine) significantly improves systemic haemodynamics, renal function and sodium Excretion in non azotemic cirrhotic patients with tense ascites, Midodrine (an alpha adrenergic agonist) has been used to improve renal haemodynamics in cirrhotics with ascites. In these patients, it increases effective arterial blood volume by causing splanchnic vasoconstriction and improves renal perfusion and glomerular filtration [18]. 
Eighty cirrhotic patients with refractory ascites attending the liver and gastroenterology unit in assiut university hospital were prospectively included in the study.

The patients were randomly assigned to two groups, standard medical treatment $(\mathrm{P}=37)$ and midodrine in maximum tolerable dose plus standard medical treatment $(\mathrm{P}=41)$.

Our study showed significant increase in mean arterial pressure in the midodrine group at 1 month with the maximum tolerable dose $(\mathrm{P}=0.04)$ but it did not change significantly in the standard medical therapy group. This was agreed by Kalambokis et al., 2007; Tandon et al., 2009 and Singh et al., 2012. [15]. [18]. [19].

Our study did not show significant change regarding heart rate $(\mathrm{P}=0.97)$. However, Kalambokis et al., 2007 recorded significant decrease in heart rate with treatment with midodrine for 7 days. [19].

In SMT group, there was no significant decrease in the patients' weight after 1 month compared to values before treatment, further more in the midodrine group we observed a significant decrease in body weight after 1 month treatment with maximum tolerable dose $(\mathrm{P}=0.00)$. This was agreed with (Mokdad et al., 2014). [16].

The urine output was significantly higher in the midodrine group after treatment $(\mathrm{p}=0.00)$ but not in the standard medical therapy group. This was agreed by Singh et al., 2012. [18].

There was no significant change in serum sodium values in the both groups after treatment. However this was disagreed with Singh et al., 2012 who noted significant decrease in serum sodium with SMT after 1 and 3 months of treatment. [18].

No significant differences between standard medical therapy and midodrine groups were observed between values for serum creatinine after treatment. This was the situation in the study done by Tandon et al., 2009 and Singh et al., 2012. [15] [18].

The present study did not show significant change in liver function tests in both groups compared with baseline values. The same situation was found with Singh et al., 2012. [18].

Baseline MELD scores were similar in both treatment groups $(p=0.14)$ and did not differ significantly from baseline in both groups after treatment for 1 month. That was not the situation with the pilot study done by. They recorded a significant deterioration in the MELD score during treatment with midodrine Tandon et al., 2009. [15].

In the present study there was no significant $\mathrm{P}$ value $(\mathrm{P}=$ 0.11 ) regarding both the frequency and volume of ascetic fluid drained after 1 month despite the improvement of mean arterial blood pressure in both the midodrine group and the SMT. This was disagreed with Sourianarayanane et al., 2011 and Singh et al., 2012. This may be explained by the short time of the study. [17] [18].

During treatment with midodrine, no side effects has been recorded that was not the same with Singh et al., 2012 who recorded pain abdomen in 6 patients of 59 patients during treatment with midodrine for 6 months. However, all episodes were mild and did not require discontinuation of therapy. [18].
In conclusion, the results of this randomized pilot study suggest that midodrine plus standard medical therapy improves systemic hemodynamics and better controls ascites without any renal or hepatic dysfunction in patients with refractory or recurrent ascites with cirrhosis as compared to standard medical therapy alone.

\section{Conclusion}

In conclusion, midodrine is a safe treatment for patients with liver cirrhosis and its addition to standard medical treatment including [low sodium diet + diuretic therapy (loop diuretic in a dose $40-160 \mathrm{mg} /$ day and distal acting diuretic in a dose $100-400 \mathrm{mg} /$ day + large volume paracentesis as needed] are associated with better control of ascites.

Recommendation, is to use midodrine in appropriate patients with ascites for appropriate time and duration with SMT may reduce the need for LVP, thus decreasing the associated risks and improving patients' quality of life.

\section{References}

[1] Sussman AN. and Boyer TD. (2011): Management of Refractory Ascites and Hepatorenal Syndrome. Curr Gastroenterol Rep. 13: 17-25.

[2] Planas R, Montoliu S, Balleste B et al., (2006): Natural history of patients hospitalized for management of cirrhotic ascites. Clin. Gastroenterol. Hepatol. 4: 1385-94.

[3] Salerno F, Cammà C, Enea M, Rssle M, Wong F. (2007): Transjugular intrahepatic portosystemic shunt for refractory ascites: a meta-analysis of individual patient data. Gastroenterology. 133: 825-34.

[4] Kashani A, Landaverde C, Medici V, Rossaro L. (2008): Fluid retention in cirrhosis: pathophysiology and management. QJM. F; 101: 71-85.

[5] Cardenas A, Arroyo V. (2003): Mechanism of water and sodium retention in cirrhosis and the pathogenesis of ascites. Best Pract Res Clin Endocrinol Metab. 17: 607-622.

[6] Runyon BA. (2002): Ascites and spontaneous bacterial peritonitis. In: Feldman M, Friedman LS, Sleisenger MH and Fordtran's gastrointestinal and liver disease. Philadelphia: saunders. p. 1517-1542.

[7] Uriz J, Gines P, Cardenas A, Sort P, Jimenez W, Salmeron JM, et al., (2000): Terlipressin plus albumin infusion: an effective and safe therapy of hepatorenal syndrome. J Hepatol. 33: 43-48.

[8] Moreau R, Asselah T, Caudat B, De Kerguenec C, Pessione F, Bernard B, et al., (2002): Comparison of the effect of terlipressin and albumin on arterial blood volume in patients with cirrhosis and tense ascites treated by paracentesis: a randomized pilot study. Gut. 50: 90-94.

[9] Ortega R, Gines P, Uriz J, Cardenas A, Calahorra B, De Las HD, et al., (2002): Terlipressin therapy with and without albumin for patients with hepatorenal syndrome: results of a prospective, nonrandomized study. Hepatology. 36: 941-948. 
[10] Singh V, Kumar R, Nain CK, Singh B, Sharma AK. (2006): Terlipressin versus albumin in paracentesis-induced circulatory dysfunction in cirrhosis: a randomized controlled trial. J Gastroenterol Hepatol. 21: 303-307.

[11] Appenrodt B, Wolf A, Grunhage F, Trebicka J, Schepke M, Rabe $C$, et al., (2008): Prevention of paracentesis-induced circulatory dysfunction: midodrine vs. albumin. A randomized pilot study. Liver Int. 28: 1019-1025.

[12] Krag A, Moller S, Henriksen JH, Niels-Henrik HR, Finn L. (2007): Terlipressin improves renal function in patients with cirrhosis and ascites without hepatorenal syndrome. Hepatology. 46: 1863-1871.

[13] Kramer, Robert E, Sokol, Ronald J, Baruch Y, Liu, Edwin, MacKenzie, Todd, Hoffenberg, Edward J, Narkewicz, Michael $R$. (2014): Large-Volume Paracentesis in the Management of Ascites in Children. Journal of Pediatric Gastroenterology \& Nutrition. 33: 245-249.

[14] Lin YF, Wang JY, Denq JC, Lin SH. (2003): Midodrine improves chronic hypotension in hemodialysis patients. Am J Med Sci. 325: 256-261.
[15] Tandon P, Tsuyuki RT, Mitchell L, Hoskinson M, Ma MM, Wong WW, et al., (2009): The effect of 1 month of therapy with midodrine, octreotide-LAR and albumin in refractory ascites: a pilot study. Liver Int. 29: 169-174.

[16] Mokdad AA, Lopez AD, Shahraz S, Lozano R, Mokdad AH, Stanaway J, Murray C JL and Naghavi M. (2014): Liver cirrhosis mortality in 187 countries between 1980 and 2010: a systematic analysis. BMC Medicine. 12: 145.

[17] Sourianarayanane A, Barnes DS, McCullough AG. (2011): Beneficial Effect of Midodrine in Hypotensive Cirrhotic Patients with Refractory Ascites. Gastroenterology \& Hepatology. 7; 189-198.39.

[18] Singh V, Dhungana SP, Singh B, Vijayverghia R, Nain CK, Sharma N, Bhalla A, Gupta PK. (2012): Midodrine in patients with cirrhosis and refractory or recurrent ascites: A randomized pilot study. Journal of Hepatology. 56: 348-354.

[19] Kalambokis G, Fotopoulos A, Economou M, Pappas K, Tsinos $E V$. (2007): Effects of a 7 day treatment with midodrine in nonazotemic cirrhotic patients with and without ascites. J Hepatol. 46: 213-221. 